CHAPTER 1

OBSERVATIONS OF SOLAR OSCILLATIONS 


\title{
Helioseismology from the South Pole The 1984/85 campaign
}

\author{
B. GELLY; E. FOSSAT; G. GREC; M. POMERANTZ \\ Département d'Astrophysique de I'I.M.S.P. \\ Universite de NICE \\ Parc Valrose \\ $F-06034$ NICE-CEDEX
}

ABSTRACT. More than 700 hours of full disk line of sight solar velocity have been recorded at the Geographic South Pole between late november 1984 and early february 1985. This paper presents very briefly some preliminary result of the analysis of this data.

Between November 27, 1984 and February 1, 1985, almost continuous data have been recorded at the geographic south pole with our sodium cell full disk spectrophotometer, in which 704 hours have been selected for analysis. That makes a duty cycle of about 45\%, which itself makes of this season a reasonably good one. A complete week of extremely good sky was missed, we could almost say as usually, as the beginning of the season before we could be ready.

The figure 1 displays part of the power spectrum of this data, taken as one single time series. It is quite clearly affected by the wind ow function (Figure 2). However, it is also quite clearly of

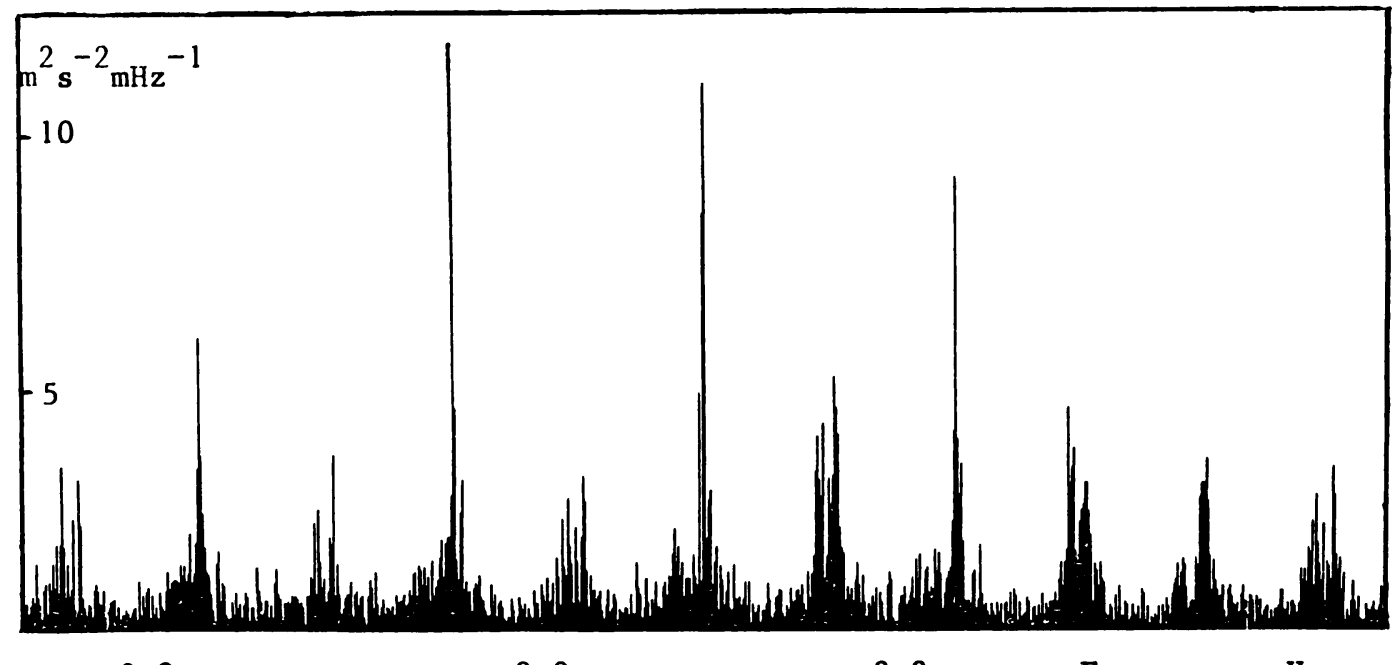

2.8

3.0

3.2

Frequency $\mathrm{mHz}$

Figure 1. Central part of the p-mode power spectrum computed from more than 700 hours of full disk velocity recorded at the South Pole between late november 84 and early february 85.

J. Christensen-Dalsgaard and S. Frandsen (eds.), Advances in Helio- and Asteroseismology, 21-23.

(c) 1988 by the IAU. 


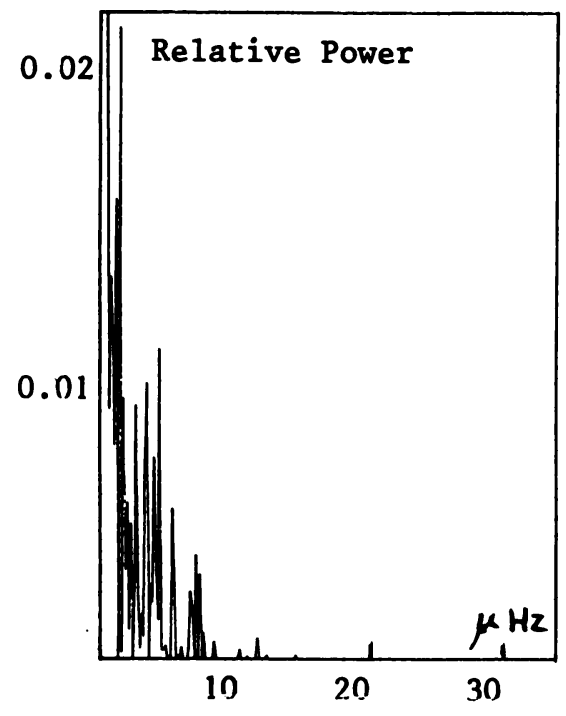

Figure 2. Power spectrum of the window function of the 1984/85 south pole data. It clearly illustrates the strong need for the setup of international networks, because that was a real good season at the south pole.

slightly lower quality than other velocity power spectra which have been published previously, including ours from the first south pole campaign (Grec et al, 1983). This is due to some technical difficulty that was identified (control of the photoelastic modulator) but that it was not possible to repair on the site. This quality is still good enough for some further analysis, and specially for making some useful comparison with previous results.

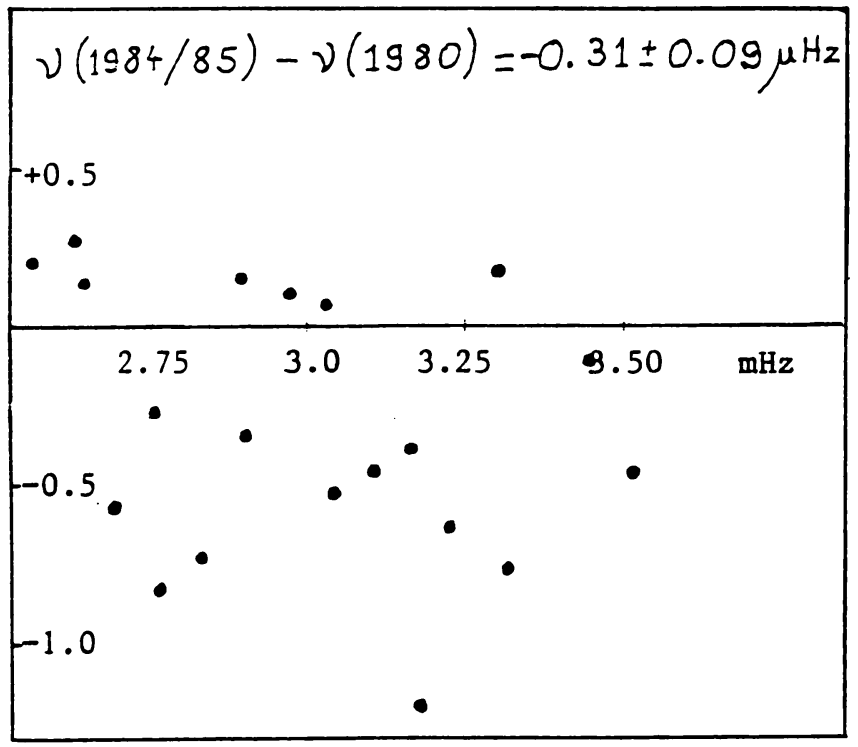

Figure 3. Comparison between $p$-mode frequencies obtained from SMM in 1980 and from the presently analysed south pole data set. This comparison seems to confirm a slight decrease of p-mode frequencies over this time interval. 
The complete analysis of this data is not finished at the time of this symosium. The figure 3 is a comparison of the p-mode frequencies measured here with the same frequencies obtained from the SIM-ACRIM data in 1980 (Woodard and Hudson, 1983). As it has been pointed out several times now, e.g. in the two here above referenced papers, the mean width of individual peaks in the five minute range is of the order of 1 or $2 \mu \mathrm{Hz}$. That explains why the scatter of individual points on this figure 3 is of the order of $0.4 \mu \mathrm{Hz}$. It simply means that it is difficult to locate more precisely the centroids of peaks without having an infinite duration of data. In the case of SIMI-ACRIM data of 1980, the duration (10 months) was significantly closer to infinity than in any other observation. However, the poorer intrinsic signal to noise ratio made the precision of the same order in determining the centroid frequencies. It must also be pointed out that in such comparison of individual frequencies, it is essentially useless to use all the high frequency part (above, say, $3.4 \mathrm{mHz}$ ) of the p-mode spectrum because in this range, the peak width increases so dramatically (Grec et al, 1983: Isaak, 1985) that the uncertainty in centroid location becomes too much larger than the small possible frequency change that we are looking for.

\section{References.}

55

Grec, G. : Fossat, E. and Pomerantz, M. 1983, Solar Phys. 82.

Woodard, M. and Hudson, H. 1983, Nature, 305, 589

Woodard, M. and Noyes, R.B. 1985, Seismology of the Sun and the Distant Stars, p303, Reidel, D.0.Gough, ed. 